\title{
La référence temporelle au futur dans les bulletins météo en France et au \\ Québec : regard variationniste sur l'oral préparé
}

Hélène Blondeau et Emmanuelle Labeau

\section{Résumé}

La distribution des variables utilisées pour l'expression de l'ultériorité a fait l'objet de nombreuses études, centrées sur l'oral conversationnel ou l'écrit. Cet article apporte un éclairage inédit sur la question en considérant les marqueurs du futur dans un contexte communicatif d'oral préparé (le bulletin météo télévisé), et dans une optique diatopique (comparaison d'un corpus français et d'un corpus québécois). L'analyse distributionnelle indique une distribution des variables spécifique au contexte de communication. Par ailleurs, l'analyse multivariée par Goldvarb X révèle une variation diatopique et l'influence de certains facteurs linguistiques, en particulier celui du type de verbe, de même que des contraintes particulières pour les deux communautés étudiées.

Mots-clés: futur synthétique - futur analytique - présent à valeur de futur - variation diatopique - bulletin météo - 
The distribution of variants used to express future temporal reference has been the object of many studies, focused on conversational speech or on written data. This article sheds new light on the issue by studying future markers in a communicative setting which consists of prepared speech (the televised weather forecast) from a diatopic perspective (comparison of French and Québécois corpora). The distributional analysis points to a distribution of variants specific to this conversational setting. Furthermore, the Goldvarb Commented [LE2]: discursive? $\mathrm{X}$ multivariate analysis reveals diatopic variation and the influence of some linguistic factors, most notably the type of verb, as well as the effect of constraints specific to the two speech communities under study.

\section{Keywords: synthetic future - analytical future - futurate present - diatopic variation} - weather forecast -

\section{Introduction}

L'usage de multiples formes verbales pour référer à un événement futur en français a déjà fait couler beaucoup d'encre. De nombreux travaux ont non seulement délimité les contextes d'usage de chaque variable tant au niveau linguistique que social (entre autres Söll 1983, Emirkanian et Sankoff 1985, Jeanjean 1988, Halmøy 1993, Vet 1993, Bahloul et Waugh 1996, Poplack et Turpin 1999), mais ils ont également discuté le remplacement potentiel du futur simple par le futur périphrastique en diachronie (Söll 1983, Blondeau 2006, Poplack et Dion 2009, Lindschouw 2010, Wagner et Sankoff 2011). 
Les études de corpus se sont concentrées sur l'écrit et l'oral conversationnel; on gagnerait donc à examiner d'autres canaux et genres. Nous abordons ici une situation de communication d'oral préparé (ou d'écrit oralisé) mâtinée de potentielle improvisation, telle qu'elle se présente dans les bulletins météo télévisés. ${ }^{1}$ De plus, alors que la plupart des travaux ont adopté l'approche sociolinguistique classique ancrée dans une communauté donnée, peu d'études ont comparé l'usage à l'aune de la variation diatopique. Dans cet article, nous souhaitons contribuer à combler cette lacune en comparant la même situation de communication en France et au Québec. Plus particulièrement, nous tenterons de répondre aux questions de recherche suivantes:

(1) Quelles sont les contraintes linguistiques déterminant le choix des formes?

(2) Parmi les facteurs extra-linguistiques, la diatopie influence-t-elle la sélection des variables exprimant la référence au futur?

(3) Peut-on établir un parallèle entre la variation dans l'oral préparé (ou l'écrit oralisé) des bulletins météo et dans les productions écrites ou orales spontanées?

1 «Il ne suffit pas à mes yeux de réciter le texte préparé. Je veux comprendre et qu'on m'explique le pourquoi des choses. Un service spécial a été créé à l'usage des médias. On y obtient renseignements, précisions et synthèse, parce qu'on n'est pas crédible si l'on ne sait pas », affirme Alain Gillot-Pétré. [...] Il a fallu que j'apprenne à être debout, à savoir quoi faire de mes mains, à improviser. Au début, ce n'était pas bon. J'ai tout réappris », raconte-t-il. (James 2014) 
Après un bref rappel de la problématique de la référence temporelle au futur (section 2), le corpus utilisé ainsi que la méthodologie adoptée seront présentés (section 3). Les résultats d'analyses distributionnelles et multivariées seront détaillés (section 4) pour aboutir à une interprétation des données mettant en relation la situation observée dans les bulletins météo avec celle de l'oral spontané à l'échelle de la francophonie.

\section{Problématique}

Le français dispose de plusieurs formes verbales pour référer à des événements ultérieurs à l'énonciation: une forme synthétique (FS) (le futur simple), une forme analytique (FA) (connue sous les noms de futur proche ou de futur périphrastique) et le présent à valeur de futur $(\mathrm{P})$. Nous allons considérer dans cette section leur origine et leurs contextes d'emploi tels qu'ils sont présentés dans les études existantes.

La forme synthétique (saurai) apparaît en latin vulgaire et se retrouve dans les plus anciens vestiges du français, comme l'illustre cet extrait des Serments de Strasbourg (842):

(1) "Pro Deo amur et pro christian poblo et nostro commun saluament, d'ist di in auant, in quant Deus sauir et podir me dunat, si saluarai eo cist meon fradre Karlo, et in adiudha et in cadhuna cosa si cum om per dreit son fradra saluar dist, in o quid il mi altresi fazet. Et ab Ludher nul plaid nunquam prindrai qui meon uol cist meon fradre Karle in damno sit."

La forme analytique (vais savoir) émerge durant le passage de l'ancien au moyen français. Les spécialistes datent son apparition du 13 ème (Poplack 2001: 415) ou du 14 ème siècle (Lindschouw 2010) ; la périphrase se diffuse indéniablement au 15 ème (Gougenheim 
1929/1971: 98, Wilmet 1970: 191, Fleischman 1982: 82ss,), et pénètre dans la langue polie et littéraire des $16^{\text {ème }}-17^{\text {ème }}$ selon Fleischman (1982: 82).

Le présent à valeur de futur est ancien comme l'atteste sa mention dans les grammaires de la première période (1500-1699) étudiées par Poplack et Dion (2009):

Notez en passant que nous avons une façon de parler où nous mettons le présent de l'indicatif pour le futur, qui est il est demain fête, quel jour est-il demain, etc (Oudin 1640: 185, cité par Fournier 2001: 4)

Ces attestations font certainement foi d'un emploi antérieur compte tenu du décalage entre usage et enregistrement par les instances normatives. Si des exemples de ce délai sont patents en ce qui concerne plusieurs cas de réforme orthographique portant sur les terminaisons verbales, ${ }^{2}$ des écarts du même type se manifestent dans le choix même de la

${ }^{2}$ À titre d'exemple, la réforme orthographique qui consiste à écrire « ai » plutôt que « oi », notamment pour les imparfaits (-ais, ait, -aient au lieu de -ois, -oit, -oient), a été préconisée par quelques grammairiens et auteurs au XVIIème siècle (Bérain, Milleran), puis au XVIIème (de Vallemont, Girard) mais elle a surtout été promue par Voltaire. En 1734, il orthographie «Français » et « Anglais » dans les « Lettres philosophiques » et, en 1752, dans «Le siècle de Louis XIV », il orthographie les imparfaits en «ai » et non en « oi ». Il a argumenté régulièrement en faveur de ce changement qui rencontre de nombreuses oppositions, comme celle de d'Alembert. La nouvelle orthographe se répand néanmoins dans les livres et est consacrée dans le «Dictionnaire critique » de Féraud en 1787. Les éditeurs Didot l'adoptent en 1798, 
morphologie verbale comme le révèlent les travaux sur la compétition du conditionnel et de l'indicatif dans les constructions hypothétiques en si (LeBlanc 2011), ou sur la concurrence entre l'indicatif et le subjonctif dans les contextes appelant le subjonctif (Poplack, Lealess et Dion 2013).

La variation dans l'expression du futur a attiré une attention considérable. C'est particulièrement le cas pour les variétés de français pratiquées en Amérique du Nord, notamment dans des données recueillies à Montréal (Emirkanian et Sankoff 1985, Zimmer 1994, Blondeau 2006, Wagner et Sankoff 2011), en Acadie (Chevalier 1994, King et Nadasdi 2003, Comeau 2014), dans la région d'Ottawa-Hull (Poplack et Turpin 1999) et à Québec (Deshaies et Laforge 1981). Plus généralement, les données conversationnelles ont été privilégiées par les études de variation dans l'expression du futur (Söll 1983, Sundell 1991, Halmoy 1993, Jeanjean 1988), ainsi que la presse écrite $\left({ }^{*}\right.$ Lesage 1991 , *Lesage et Gagnon 1992, Waugh et Bahloul 1996, Wales 2002).

La plupart des études se sont centrées sur les nuances qui distinguent les formes synthétique et périphrastique. Alors qu'on a longtemps expliqué la différence en fonction d'une distinction temporelle-proximité pour le FA, éloignement pour le FS—face à l'événement futur, les études sur le français québécois ont montré que ce facteur ne jouait pas de rôle mais qu'une série d'autres éléments contraignaient la variation. Le constat s'avère toutefois différent dans les études sur le français acadien, une variété réputée plus

mais l'Académie française ne la rendra officielle qu'en 1835 (Brunot, F. 1966: 961963).

${ }^{3}$ L'astérisque indique les études canadiennes. 
conservatrice (Comeau 2014). L'idée bien ancrée d'une différentiation temporelle entre un futur proche (FA) et un futur distant (FS) doit donc être soumise à l'analyse.

La distinction selon la distance temporelle remonte à la Grammaire de Port Royal (1660) dans laquelle une correspondance avait été établie entre le Paulopost futurum grec et le FA. La forme périphrastique avait donc été baptisée futur prochain par l'Abbé Antonini dans ses Principes de la grammaire françoise, pratique et raisonnée (1753). Le concept de proximité n'en perdure pas moins et il est toujours utilisé par Confais (1995) ou au moins invoqué sous une forme atténuée chez Fleischman (1982) ou Jeanjean (1988). Un examen des recommandations des grammaires prescriptives s'étalant sur plus de cinq siècles (Poplack et Dion 2009) indique cependant une absence de consensus quant aux facteurs qui expliqueraient le choix d'une forme au détriment de l'autre.

Dans les études contemporaines de la référence au futur, la distinction entre le FS et le FA repose sur une variété de traits temporels, modaux, discursifs, grammaticaux et sociolinguistiques. On relève parmi les traits temporels: la distance temporelle (Poplack et Turpin 1999, Poplack 2001, King et Nadasdi 2003, Blondeau 2006), l'imminence (Poplack et Turpin 1999, Poplack 2001, King et Nadasdi 2003), et la référence temporelle (Grimm 2010). Du côté modal, on a évoqué l'impact de la polarité négative sur l'emploi du FS (Poplack et Turpin 1999, Poplack 2001, King et Nadasdi 2003, Blondeau 2006, Grimm 2010), le rôle du degré de certitude attaché au procès verbal (King et Nadasdi 2003, Blondeau 2006, Grimm 2010), et la présence d'indications de contingence, particulièrement marquée par si et quand (Poplack et Turpin 1999, Poplack 2001, King et Nadasdi 2003, Blondeau 2006, Grimm 2010). Parmi les facteurs discursifs, la présence de spécifications adverbiales (Poplack et Turpin 1999, Poplack 2001, King et Nadasdi 2003, 
Blondeau 2006, Grimm 2010), notamment de fréquence (Poplack 2001), a été jugée discriminante. D’un point de vue grammatical, des caractéristiques du sujet telles que la personne (Poplack et Turpin 1999, Poplack 2001, King et Nadasdi 2003, Grimm 2010) et le nombre (Poplack 2001, Blondeau 2006), ainsi que du verbe (types de verbes, particulièrement les états - Poplack 2001) ont été mentionnées. Finalement, des facteurs stylistiques comme le niveau de formalité (Jeanjean 1988, Grimm 2010, Mougeon et al. 2010) ou des facteurs sociaux (Blondeau 2006; Grimm 2010; Blondeau, Dion et Ziliak 2014) semblent entrer en ligne de compte.

S’il est généralement accepté que le FA est en train de prendre le pas sur le FS, plusieurs variables paraissent maintenir ce dernier. La polarité semble la variable la plus remarquable compte tenu de l'affinité entre le FS et la négation. En contextes positifs, la contingence favorise le FS (Blondeau 2006). ${ }^{4}$ Le FS est préféré dans le discours rétrospectif (Jeanjean 1988: 236):

(2) Cette fois-ci ce sera une double faute

Sur ce match la balle ne reviendra pas

C'est Boris qui accélérera le premier et qui marquera le point

D'un point de vue sociolinguistique, le FS semble favorisé dans la langue surveillée et en relation avec des thèmes formels (Mougeon et al. 2010). Par ailleurs, un déclin du FS paraît se manifester parmi les jeunes (Zimmer 1994), ce qui montrerait un changement en cours

\footnotetext{
${ }^{4}$ Par exemple, dans le cas d'occurrences dont le contexte porte des marques d'incertitude
} face à l'événement futur comme dans les propositions introduites par si ou par quand ou des formulations comme peut-être ou on le sait pas. 
au niveau communautaire. ${ }^{5}$ Cependant, l'usage du FS semble se conserver, ou même augmenter chez certains locuteurs au fur et à mesure qu'ils vieillissent, ce qui pourrait indiquer, soit un retranchement face à la tendance communautaire, soit simplement un effet de gradation d'âge en lien avec une histoire sociolinguistique qui amènerait certains locuteurs à intégrer davantage les formes légitimes dans leur parler (Blondeau 2006, Wagner et Sankoff 2011).

Par contre, la certitude présente des affinités avec le FA (King et Nadasdi 2003: 330, Grimm 2010: 87). Celui-ci se combine à la première personne (King et Nadasdi 2003: 330, Grimm 2010: 86) alors que le FS corrèle avec vous (Grimm 2010: 86).

Les études qui incluent le présent à valeur de futur tendent à le rapprocher du FA et l'interprètent comme une garantie de la valeur de véracité de la proposition (Confais 1995: 397) et comme une indication de la confiance de l'émetteur dans la menée à bien du procès (Imbs 1968) puisque les conditions de réalisation sont déjà remplies au moment de la parole (Vet 1993: 75). Poplack et Turpin (1999) suggèrent que, vu l'appropriation des valeurs du FS par le FA, le présent à valeur de futur pourrait remplir les anciennes valeurs du FA en s'associant avec des adverbiaux temporels spécifiques.

Après ce bref survol de la problématique, nous présenterons dans la section suivante le corpus inédit sur lequel se base l'étude avant de décrire la méthode d'analyse utilisée.

\section{Choix du corpus et méthodologie}

\subsection{Le corpus}

\footnotetext{
${ }^{5}$ Mais voir à l'opposé la vitalité du FS dans le texto (Labeau 2014).
} 
La présente étude se distingue des précédentes à la fois par la nature des données étudiées et par sa dimension diatopique. Elle compare en effet la référence au futur dans deux variétés de français en s'appuyant sur un corpus de bulletins météo diffusés à la télévision en France et au Québec.

L'examen d'un tel corpus présente plusieurs avantages. Tout d'abord, comme ces bulletins météo appartiennent au domaine public, il s'avère facile d'y accéder et de les recueillir. Par ailleurs, ce genre de données est diatopiquement comparable puisque la formule du bulletin météo télévisé se retrouve dans les deux communautés francophones visées: cet événement de communication présente indubitablement des caractéristiques indépendantes de la géographie. Par ailleurs, le contexte référentiel particulier aux bulletins météo se prête bien à l'émergence de différentes formes du futur puisque les présentateurs ont pour tâche principale de fournir des prévisions météorologiques impliquant par définition une référence temporelle à un moment postérieur. Enfin, ce type de données, se distinguant tant de l'oral spontané généralement recueilli en sociolinguistique que de l'écrit journalistique, élargit la prise en compte de la variation à de nouveaux contextes situationnels. En effet, bien qu'il puisse y avoir une certaine part d'improvisation, nous sommes en présence d'un oral préparé (ou d'un écrit oralisé), un genre de situation susceptible d'influencer la variation.

Le corpus analysé consiste en 24 bulletins météo télévisés : 12 bulletins de la France diffusés sur trois chaînes nationales (TF1, France 2 et France 3) et 12 bulletins du Québec diffusés sur la chaîne Radio-Canada. Le recueil des bulletins français s'est échelonné de la fin du mois de décembre 2011 au début janvier 2012 alors que celui des bulletins québécois a eu lieu durant la seconde moitié du mois de janvier 2012. Les bulletins ont 
ensuite été transcrits orthographiquement. Le corpus totalise près d'une heure d'enregistrement pour un peu moins de 13000 mots. Le bulletin québécois dure en moyenne 2 minutes 44 pour 608 mots (SD : 49,53) alors que le bulletin français moyen est long de 2 minutes 17 et compte 464 mots (SD : 224,62). ${ }^{6}$ La plus grande homogénéité du corpus nord-américain s'explique par le fait que tous les bulletins sont des bulletins du soir sur une même chaîne. Par contre, le corpus européen se compose de bulletins issus de trois chaînes et diffusés soit le midi soit le soir.

\begin{tabular}{|l|l|l|l|l|l|}
\hline ed & Chaine & Date & Présentateur & Duré & Mots \\
\hline F1. & FR3 & $\begin{array}{l}\text { vendredi } 30-12-2012 \quad \text { (météo des } \\
\text { neiges) }\end{array}$ & Thomas Lerides & $0^{\prime} 50$ & 200 \\
\hline F2. & A2 & dimanche 01-01-2012 (midi) & Isabelle Martinet & $2^{\prime} 02$ & 390 \\
\hline F3. & FR3 & dimanche 01-01-2012 (soir) météo des & Thomas Lerides & $1^{\prime} 08$ & 237 \\
\hline F4. & A2 & lundi 02-01-2012 (midi) & & & \\
\hline F5. & TF1 & lundi 02-01-2012 (midi) & Laurent Romejko & $3^{\prime} 35$ & 768 \\
\hline F6. & FR3 & lundi 02-01-2012 (soir) - météo des & Thomas Lerides & $0^{\prime} 50$ & 184 \\
\hline
\end{tabular}

6 TF1 3’13, 629 mots (SD : 149,46); A2: 2’40, 552 mots (SD : 71,73); FR3 0’59 / 210 mots (SD : 22,99). 


\begin{tabular}{|c|c|c|c|c|c|}
\hline F7. & $\mathrm{A} 2$ & mardi 03-01-2012 (soir) & Laurent Romejko & $1 ' 58$ & 441 \\
\hline F8. & A2 & mercredi 04-01-2012 (midi après JT) & Isabelle Martinet & $3 ’ 02$ & 609 \\
\hline F9. & TF1 & mercredi 04-01-2012 (midi) & Evelyne Dhéliat & $3 ’ 27$ & 690 \\
\hline F10. & TF1 & mercredi 04-01-2012 (soir) & Evelyne Dhéliat & $2 ’ 27$ & 474 \\
\hline F11. & TF 1 & jeudi 05-01-2012 (midi) & Evelyne Dhéliat & $2 ’ 50$ & 544 \\
\hline F12. & FR3 & 05-01-2012 (soir) météo des neiges & Femme non identifiée & $1 ' 10$ & 219 \\
\hline & & & & $27^{\prime} 24$ & 5564 \\
\hline
\end{tabular}

Tableau 1: Description des 12 bulletins français

\begin{tabular}{|c|c|c|c|c|c|}
\hline Code & Chaine & Date & Présentateur & Durée & Mots \\
\hline Q1. & $\mathrm{RC}$ & Mardi 10-01-2012 (22h) & Pascal Yiacouvakis & 2’49 & 645 \\
\hline Q2. & $\mathrm{RC}$ & Mercredi 11/01/2012 (22h) & Pascal Yiacouvakis & $2 ’ 40$ & 671 \\
\hline Q3. & $\mathrm{RC}$ & Jeudi 12-01-2012 (22h) & Pascal Yiacouvakis & $2 ’ 33$ & 608 \\
\hline Q4. & $\mathrm{RC}$ & Vendredi 13-01-2012 (22h) & Josiane Cuierrier & 2’46 & 619 \\
\hline Q5. & $\mathrm{RC}$ & Mardi 17-01-2012 (22h) & Pascal Yiacouvakis & $2 ’ 30$ & 609 \\
\hline Q6. & $\mathrm{RC}$ & Mercredi 18-01-2012 (22h) & Pascal Yiacouvakis & $2 ’ 48$ & 639 \\
\hline Q7. & $\mathrm{RC}$ & Jeudi 19-01-2012 (22h) & Pascal Yiacouvakis & $2 ’ 22$ & 608 \\
\hline Q8. & $\mathrm{RC}$ & Vendredi 20-01-2012 & Josiane Cuierrier & $2 ’ 35$ & 509 \\
\hline
\end{tabular}




\begin{tabular}{|l|l|l|l|l|l|}
\hline Q9. & RC & Mardi 24-01-2012 & Pascal Yiacouvakis & 2'30 & 552 \\
\hline Q10. & RC & Mercredi 25-01-2012 & Pascal Yiacouvakis & $2 ' 37$ & 567 \\
\hline Q11. & RC & Jeudi 26-01-2012 & Pascal Yiacouvakis & $2 ' 55$ & 683 \\
\hline Q12. & RC & Vendredi 27-10-2012 & Josiane Cuierrier & $2 ' 45$ & 590 \\
\hline & & & & $31 ' 50$ & 7300 \\
\hline
\end{tabular}

Tableau 2: Description des 12 bulletins québécois

Bien que la majorité des travaux antérieurs se soient centrés sur la paire FS/FA, nous avons opté, dans un premier temps, pour un relevé plus exhaustif de la référence temporelle au futur. En effet, il nous semblait crucial de relever l'ensemble des formes pour bien documenter le contexte situationnel à l'étude. En conséquence, l'inventaire des formes verbales comprend non seulement la forme synthétique (3) et la forme analytique (4) comme variantes de prédilection de l'oral spontané, mais aussi le présent (5) dans la mesure où l'énoncé réfère à un événement futur. Nous avons également choisi d'inclure dans l'analyse préliminaire le conditionnel car il nous apparaissait comme une variante possible pour indiquer un événement futur (6). Ceci dit, les conditions d'émergence des formes conditionnelles mériteraient un examen plus approfondi dans une étude ultérieure. Enfin, nous avons choisi d'extraire aussi les formes non verbales disponibles au répertoire des locuteurs (7).

(3) voici maintenant les températures attendues cet après-midi elles seront en légère baisse, mais toujours douces pour la saison elles varieront de sept à seize degrés du 
Cantal jusqu'à l'extrême Sud du pays à Collions par exemple (France, A2, Isabelle Martinet, F8)

(4) ces précipitations vont commencer assez tard demain à peu près cinq à dix centimètres possibles ça va changer en pluie pour vendredi le matin il y aura du vent (Québec, RC, Pascal, Q2)

(5) aux Iles de la Madeleine ça demeure très venteux demain matin avec des averses de neige (Québec, RC, Pascal, Q6)

(6) il pourrait tomber cinq centimètres supplémentaires vendredi avec une chute des températures en Ontario sous forme de pluie mêlée de neige fondante vers Toronto les températures seront fortement à la baisse à partir de vendredi (Québec, R-C, Pascal, Q2)

(7) beaucoup de vent au programme au cours des prochains jours à venir (France, Antenne 2, Laurent, F4)

Ce relevé nous montre qu'en plus des trois tiroirs anticipés, on rencontre d'autres formes verbales marginales comme le subjonctif et le futur antérieur mais surtout une proportion assez importante de références non verbales à l'avenir, de l'ordre de 20 pourcent dans le corpus de France et d'un peu moins dans les données du Québec, un aspect sur lequel nous reviendrons dans nos prochaines recherches. Par souci de comparabilité avec les études antérieures, nous nous concentrerons ici sur le FS, le FA et le P et écartons les autres variantes des analyses quantitatives qui suivent.

\subsection{Méthodes d'analyse}


Les trois formes retenues pour l'analyse représentent un total de 515 occurrences dans l'ensemble du corpus France-Québec. Les analyses proposées se distinguent en deux types. Dans un premier temps, une analyse distributionnelle montre comment les variantes se répartissent dans l'ensemble du corpus afin d'identifier les tendances générales, puis dans les deux variétés étudiées de manière à donner un premier aperçu de la variation diatopique.

Dans un deuxième temps, une analyse multivariée examine le poids relatif des facteurs influençant la variation dans l'emploi des formes verbales pour permettre une comparaison avec les analyses antérieures. Pour ce faire, le contexte de chaque occurrence a fait l'objet d'un examen des facteurs d'influence potentielle sur la variation. Deux facteurs extralinguistiques sont examinés dans la présente analyse: la communauté et le genre. Quant aux six variables linguistiques retenus, ils comprennent la polarité, la distance temporelle et la présence adverbiale — facteurs abondamment discutés dans les études variationnistes antérieures-mais aussi le type de verbe, le type de sujet et la personne, qui pourraient éventuellement jouer un rôle dans l'explication de la variation.

L'étude quantitative est menée avec le logiciel Goldvarb X (Sankoff et al. 2005) qui permet de dégager dans un premier temps la distribution des formes puis de traiter les données selon une analyse multivariée (Tagliamonte 2006). L'analyse multivariée identifie quels groupes de facteurs contraignent la variation. À l'intérieur d'un groupe de facteurs significatifs, les facteurs sont rangés les uns par rapport aux autres selon leur poids relatifs. 
permet de dégager l'ordre des contraintes sur la variation. ${ }^{7}$ L'analyse permet donc de modéliser les différentes dimensions de la variation. Pour des raisons de comparabilité avec les études antérieures, nous présentons les résultats selon la valeur d'application du futur synthétique. Les tableaux qui présentent les résultats résument donc les groupes de facteurs qui contraignent le choix de cette forme verbale au détriment des deux autres.

Enfin, nous présentons deux sous-analyses selon la communauté québécoise ou française pour les comparer. Pour le sous-corpus du Québec, nous contrastons la tendance observée dans les bulletins météo avec les productions orales spontanées puisque plusieurs études antérieures ont documenté cette variété, en particulier en français montréalais (Emirkanian et Sankoff 1985, Zimmer 1994, Blondeau 2006, Wagner et Sankoff 2011).

\section{Résultats}

Dans cette section, nous procèderons à une analyse distributionnelle des variantes avant de mener des analyses multivariées.

\subsection{Analyse distributionnelle des variantes}

La section qui s'ouvre présente les résultats d'une analyse distributionnelle des formes dans le corpus. Cette première analyse indique comment les variantes se répartissent dans

\footnotetext{
${ }^{7}$ À l'intérieur d'un groupe de facteurs, l'écart se mesure à partir de la différence entre le facteur qui a le poids le plus élevé et celui qui a le poids le moins élevé, ce qui permet de hiérarchiser les groupes entre eux. Un groupe avec un écart élevé sera situé plus haut dans la hiérarchie des contraintes alors qu'un groupe avec un écart plus faible occupera une moindre place.
} 
l'ensemble des bulletins météo. Comme le montre la figure 1, le futur synthétique constitue la variante majoritaire, suivi du présent à valeur de futur puis du futur analytique. La distribution observée dans l'oral préparé que constitue le bulletin météo tranche avec l'oral spontané, d'une part, par la nette prépondérance du futur synthétique, une variante considérée en régression à l'oral et, d'autre part, par la faible place occupée par le futur analytique, une variante en progression dans le vernaculaire, particulièrement avancée au Québec. Par ailleurs, on peut s'étonner de la large part dévolue à la variante du présent à valeur de futur, une forme souvent négligée dans les analyses antérieures en raison de sa faible présence à l'oral (Blondeau 2006, Wagner et Sankoff 2011).

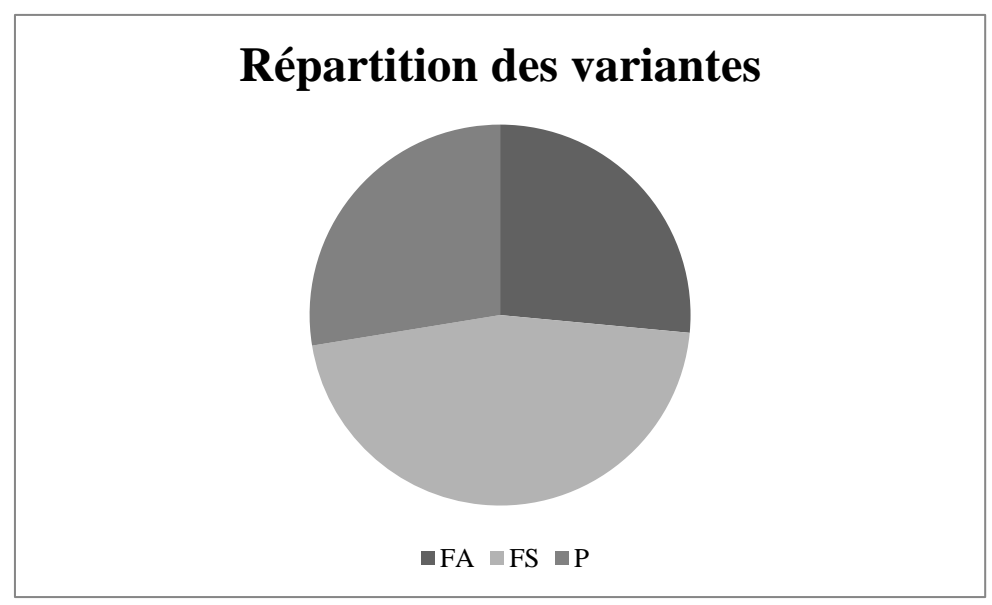

Figure 1: Les trois variantes de la référence temporelle au futur dans le corpus des bulletins météo France-Québec

Commented [PC6]: Je pense qu'il faudra que les figures soient en noir et blanc. Est-ce que ces figures en noir et blanc vous conviennent ?POUR EMMANUELLE, ok

Si on examine maintenant les variantes pour chacune des communautés, la répartition générale se confirme comme l'illustrent les résultats présentés à la figure 2 . 


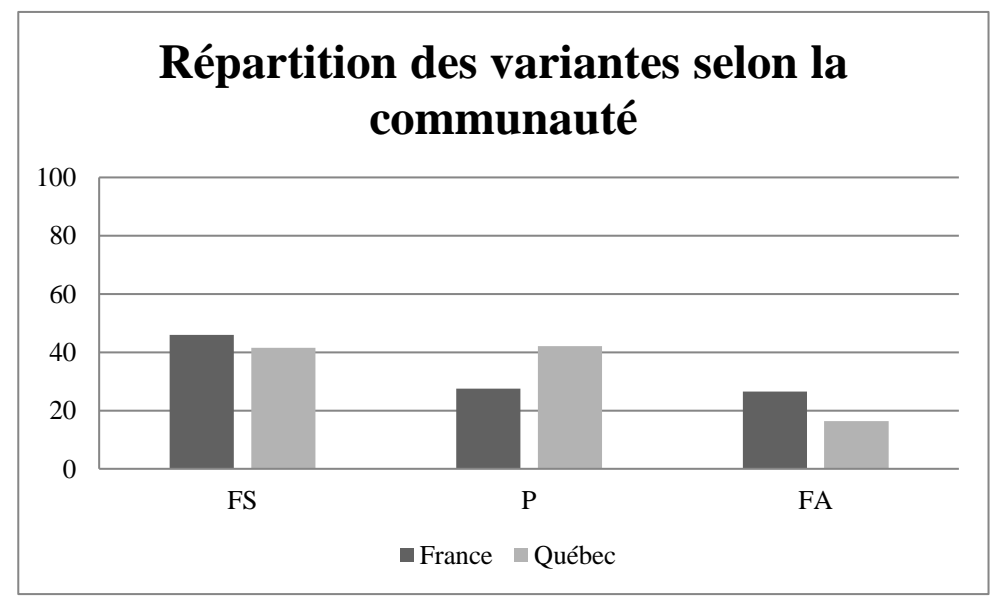

Figure 2: Répartition des variantes de la référence temporelle au futur en France et au Québec

On constate d'abord qu'en France, le futur synthétique arrive largement en tête alors que les deux autres formes sont pratiquement au coude à coude. Au Québec, le futur synthétique se situe légèrement en tête de peloton, suivi du présent à valeur de futur puis du futur analytique. Nous verrons dans quelle mesure ces différences sont significatives dans l'analyse multivariée ci-dessous. Cette distribution se distingue de la situation de l'oral spontané québécois, réputé pour la rareté du futur synthétique, considéré en déclin dans la communauté. Par ailleurs, il faut constater la forte présence du présent en comparaison avec la faiblesse de sa représentation dans l'oral spontané, un trait qui explique son exclusion de plusieurs analyses variationnistes du français québécois (Blondeau 2006, Wagner et Sankoff 2011). Il s'avère donc important de retenir cette forme pour l'analyse multivariée dans la prochaine section. Enfin, le fait que le futur analytique arrive bon dernier au Québec ne concorde pas avec l'avancée de cette forme dans l'oral spontané et l'hypothèse d'un changement en cours à l'échelle de la communauté. 
En somme, bien que le futur simple et le futur périphrastique entrent en concurrence comme dans l'oral spontané, le bulletin météo laisse davantage de place à d'autres formes, notamment l'indicatif présent qui s'avérait une variante négligeable dans les études sur l'oral spontané. Ces différences forcent à s'interroger sur le contexte situationnel particulier au bulletin météo. En effet, son contexte communicationnel, impliquant nécessairement une référence implicite au futur, pourrait expliquer que le présent convienne bien à ce genre de discours. Comme l'événement de communication du bulletin météo réfère par nature à l'ultériorité tant du point de vue de la production que de la perception, cela pourrait diminuer l'importance de la marque morphologique synthétique ou analytique sur le verbe, en quelque sorte redondante dans ce contexte. ${ }^{89}$

Pour mieux cerner la situation, nous procédons dans la section suivante à une analyse variationniste multivariée en faisant intervenir différents groupes de facteurs susceptibles de contribuer à expliquer la variation observée dans le corpus analysé.

\subsection{Analyses multivariées}

Dans un premier temps, nous présentons les résultats d'une analyse multivariée sur l'ensemble des données du corpus France-Québec, pour ensuite nous pencher plus attentivement sur chacune des communautés. Les résultats de la première analyse multivariée figurent au tableau 3. Ces résultats sont présentés selon la variante d'application du futur synthétique pour faciliter la comparaison avec les études antérieures

${ }^{8}$ Pareillement, la représentation du présent dans la référence au passé se retrouve dans de nombreux genres, notamment l'histoire (Labeau et Holyoak 2007). 
qui prennent souvent cette variante comme étalon de mesure. Les tableaux montrent les groupes de facteurs exerçant un effet significatif sur la variation. À l'intérieur de ces groupes, un facteur dont le poids relatif dépasse ,5 favorise le choix de la variante d'application, en l'occurrence le futur synthétique, alors qu'en facteur obtenant un poids relatif inférieur à ,5 la défavorise.

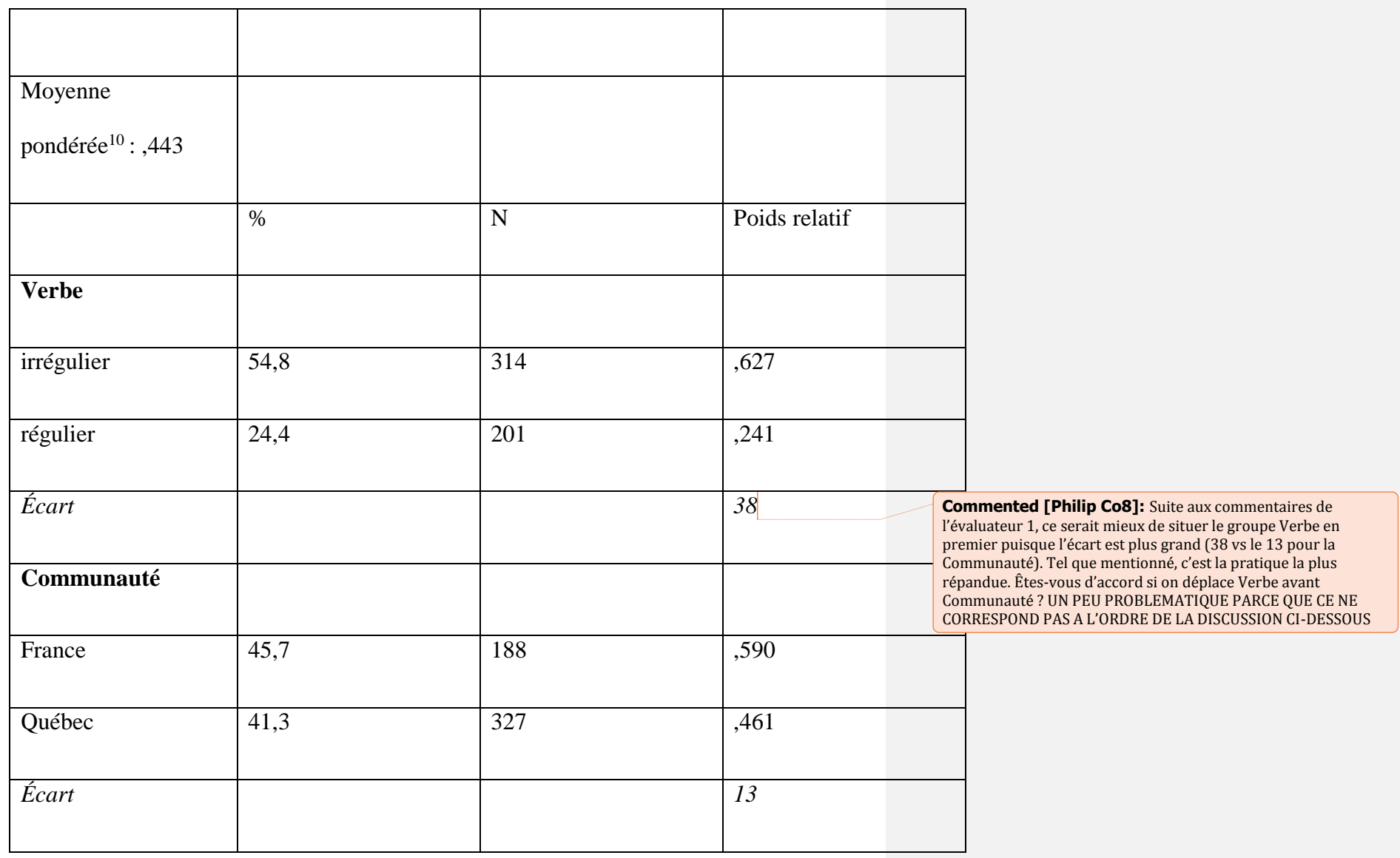

${ }^{10}$ Traduction française utilisée pour input. Cela correspond plus ou moins à la fréquence de la variante d'application. 


\section{Tableau 3: Analyse multivariée des groupes de facteurs contribuant au choix du futur synthétique dans les bulletins météo France-Québeci}

${ }^{\text {i } G r o u p e ~ d e ~ f a c t e u r s ~ r e j e t e ́ s ~ d e ~ l ' a n a l y s e: ~ p o l a r i t e ́, ~ d i s t a n c e ~ t e m p o r e l l e, ~ p r e ́ s e n c e ~}$ adverbiale, type de sujet, personne, genre

L'analyse des données indique que, des deux facteurs extralinguistiques considérés, la communauté et le genre, seul le premier a un effet. Ainsi, les bulletins météo français favorisent le futur synthétique alors que ceux du Québec en comparaison le défavorisent. Un effet diatopique entre donc en jeu. Il conviendra de vérifier si cette influence géographique se confirme en incluant d'autres communautés dans les analyses de la variation à l'échelle de la francophonie. ${ }^{11}$ Par contre, rien n'est concluant du côté de la répartition homme / femme, puisque ce groupe de facteur est rejeté du modèle statistique.

Du côté des contraintes linguistiques, le seul groupe de facteurs sélectionnés par le modèle statistique concerne le type de verbe. Ce groupe de facteurs du type de verbe contraint la variation et obtient un écart de 38 , ce qui le situe en première place dans la hiérarchie des contraintes linguistiques. Les verbes irréguliers favorisent le futur synthétique alors que les verbes réguliers le défavorisent. On peut interpréter ce résultat à la lumière de la tendance des verbes irréguliers à préférer des variantes conservatrices,

${ }^{11}$ Le corpus des bulletins météo (Labeau 2012) contient également des données pour la Suisse et la Belgique. Les premiers éléments d'une analyse combinant les quatre communautés francophones permettent d'entrevoir un effet diatopique s'appliquant aux quatre communautés (Labeau et Blondeau 2015). 
comme cela a été observé pour d'autres variables sociolinguistiques se rattachant à la morphologie verbale, notamment la rétention de la morphologie du subjonctif (Poplack 2001) ou du passé simple (Labeau 2015).

Il convient maintenant de glisser quelques mots sur certains des groupes de facteurs écartés de l'analyse statistique, notamment la polarité, la distance temporelle et la présence adverbiale, aspects souvent discutés dans les sources sur le sujet de la référence au futur. Tout d'abord, on ne constate pas d'effet de la distance temporelle face à l'événement futur, ceci confirmant la tendance de l'oral spontané contemporain. Cependant, en contraste avec les tendances observées dans l'oral spontané, on remarque l'absence d'effet de la polarité dans l'oral préparé des bulletins météo. Ceci pourrait étonner puisque qu'à l'oral spontané, tant au Québec qu'en France, les énoncés négatifs favorisent la variante synthétique, ce contexte étant devenu en quelque sorte la chasse-gardée de cette forme. Si on peut s'étonner de l'absence d'impact de la polarité, il faut néanmoins rester prudent car les énoncés négatifs demeurent rares dans les données de ce corpus, les bulletins météo traitant davantage du temps qu'il fera et non de ce qui n'adviendra pas. Enfin, la présence d'un adverbe n'exerce pas d'influence sur le choix du futur synthétique. Dans l'analyse, ni l'effet du type de sujet, ni celui de la personne ne sont sélectionnés.

Comme le facteur extralinguistique de la communauté influence significativement la variation pour l'ensemble des données, la scission du corpus en deux afin d'approfondir l'analyse se justifie. Nous présentons d'abord la situation dans les bulletins météo québécois car cette variété a été étudiée en profondeur en ce qui a trait à l'oral spontané. 
Le sous-corpus des bulletins météo québécois contient 327 occurrences. Les groupes de facteurs potentiellement susceptibles de contraindre la variation dans ce souscorpus ont fait l'objet d'une sous-analyse dont les résultats sont détaillés dans le tableau 4.

\begin{tabular}{|c|c|c|c|c|}
\hline $\begin{array}{l}\text { Moyenne } \\
\text {,374 }\end{array}$ & & & & \\
\hline & $\%$ & $\mathrm{~N}$ & Poids relatif & \\
\hline \multicolumn{5}{|l|}{ Verbe } \\
\hline irrégulier & 53,2 & 231 & ,653 & \\
\hline régulier & 12,5 & 96 & ,180 & \\
\hline Écart & & & 47 & $\begin{array}{l}\text { Commented [Philip Co9]: De nouveau, nous avons organisé } \\
\text { les groupes de facteurs selon l'écart. Est-ce que ça vous va? }\end{array}$ \\
\hline \multicolumn{5}{|l|}{ Genre } \\
\hline femmes & 53,8 & 80 & ,644 & \\
\hline hHommes & 37,2 & 247 & ,452 & Commented [LE10]: dédoublement \\
\hline Écart & & & 19 & \\
\hline \multicolumn{5}{|c|}{ Type de sujet } \\
\hline $\mathrm{SN}$ & 23,9 & 67 & ,364 & \\
\hline
\end{tabular}




\begin{tabular}{|c|c|c|c|c|}
\hline pronom & 45,8 & 260 & ,539 & Commented [LE11]: faire remonter \\
\hline Écart & & & 18 & \\
\hline Adverbe & & & & \\
\hline absence & 34,4 & 122 & ,394 & \\
\hline présence & 45,4 & 205 & ,564 & \\
\hline Écart & & & 17 & \\
\hline Distance & & & & \\
\hline hodiernal & 38,2 & 204 & ,435 & \\
\hline distant & 46.3 & 123 & ,607 & \\
\hline Écart & & & 17 & \\
\hline
\end{tabular}

-Groupes de facteurs rejetés de l'analyse: polarité

Tableau 4: Groupes de facteurs contraignant le choix du futur synthétique

dans les bulletins météo québécois

Parmi les facteurs linguistiques, on constate que le type de verbe contraint la variation de la manière la plus importante, l'écart pour ce groupe de facteur atteignant 47 . Cet effet va dans la même direction que la tendance identifiée dans l'analyse d'ensemble présentée cidessus. En outre, l'analyse révèle l'effet d'autres groupes de facteurs masqués dans l'analyse d'ensemble. Le second facteur sélectionné est le type de sujet. Les sujets pronominaux favorisent l'adoption du futur synthétique alors que les sujets nominaux la 
défavorisent. Par ailleurs, on remarque que la présence d'un adverbe encourage l'adoption de la forme synthétique, mais avec un écart moindre qui atteint 17. Quant à la distance temporelle dont l'écart s'élève à 17, elle exerce un effet sur la variation, les événements distants favorisant le choix de la forme synthétique, tandis que les éléments hodiernaux (à l'intérieur d'une période de 24 heures) et post-hodiernaux la défavorisent. Ce qui est remarquable, pour les données québécoises, est l'absence d'effet de la polarité sur la variation puisque ce facteur était considéré crucial dans les études antérieures sur le français parlé au Québec. ${ }^{12}$

À la lumière de ce tableau, des différences importantes entre l'oral spontané et l'oral préparé ressortent dans les données québécoises. La distance temporelle, non significative dans l'oral spontané, joue un rôle dans l'oral préparé, les événements à portée temporelle distante et indéterminée soutenant l'adoption de la forme synthétique. Ce résultat semble aller de pair avec la prescription des grammaires normatives. Enfin, l'absence de la polarité comme facteur significatif distingue l'oral préparé de l'oral spontané. En définitive, l'oral préparé se détacherait donc du vernaculaire local et

${ }^{12}$ Signalons cependant encore une fois qu'il y a peu d'occurrences à la forme négative. Par ailleurs, il faut noter que la polarité n'exerce pas d'effet en français acadien, une variété souvent jugée plus conservatrice, en matière de morphosyntaxe (King et Nadasdi 2003; Comeau 2014). 
l'événement de communication représenté dans les bulletins météo tendrait davantage vers la norme véhiculée par le français de référence. ${ }^{13}$

Enfin, l'analyse décèle un effet de genre. Bien qu'on puisse imputer ce résultat à la tendance—déjà bien établie en sociolinguistique—des femmes à s'orienter davantage vers les variantes normatives, il faut le relativiser, étant donné qu'il n'y a qu'un seul individu dans chacune des catégories de genre dans le corpus québécois. Ce résultat pourrait simplement constituer un épiphénomène de la production idiosyncratique. Cette influence potentielle du genre serait à vérifier à partir d'un corpus de données québécois impliquant davantage de locuteurs.

Il convient maintenant d'examiner les groupes de facteurs influençant la variation dans le sous-corpus des bulletins météo français, même si les études sur l'oral spontané en France sont beaucoup moins nombreuses. Il faut de surcroît se rappeler en examinant les résultats que cette analyse ne compte que 188 occurrences. Les résultats présentés au tableau 5 montrent que seulement deux groupes de facteurs exercent un effet significatif sur la variation.

\begin{tabular}{|l|l|l|l|}
\hline & & & \\
\hline Moyenne pondérée : & & & \\
\hline & & & \\
\hline & Poids relatif & $\mathrm{N}$ & $\%$ \\
\hline
\end{tabular}

${ }^{13}$ Cette tendance se manifeste dans le corpus de discours politiques étudiés par Labeau (2008), où le passé simple est utilisé dans la communication orale préparée. 


\begin{tabular}{|l|l|l|l|l|}
\hline Verbe & & & \\
\hline irrégulier & 59 & 83 &, 655 \\
\hline régulier & 35,2 & 105 &, 376 & \\
\hline Écart & & & 28 & \multicolumn{2}{|c|}{ Commented [Philip Co12]: De nouveau, organisé selon } \\
\hline Genre & & & & \\
\hline homme ça vous va?
\end{tabular}

Tableau 5: Facteurs contraignant le choix du futur synthétique dans les bulletins

\section{météo français}

En ce qui concerne les contraintes linguistiques, on observe que le groupe de facteurs du type de verbe exerce un effet, alors que les autres facteurs sont écartés du modèle statistique. Cet effet va dans la même direction que dans l'analyse des données québécoises, dans lesquelles les verbes irréguliers favorisaient le futur synthétique.

Enfin, en ce qui a trait au facteur extralinguistique du genre, il est sélectionné par le modèle statistique, les journalistes météo masculins favorisant la variante synthétique. Signalons toutefois que cet effet s'exerce dans le sens contraire de ce qui avait été observé au Québec, un fait qui nous invite à la prudence dans l'interprétation des données, surtout vu le nombre limité de locuteurs impliqués, qui pourrait laisser place à des effets idiosyncratiques. 


\section{Conclusion}

Cette recherche s'est penchée sur la référence temporelle au futur en français, en élargissant l'analyse à des données d'un genre inédit, celui des bulletins météo, un événement de communication représentant un oral préparé (ou un écrit oralisé). Nos questions de recherche incluaient des contraintes sociales comme la diatopie et le genre, des contraintes linguistiques sur la sélection des variables exprimant la référence au futur, et nous allons revenir sur chacun de ces points. Par ailleurs, l'analyse de la distribution des formes porte à s'interroger sur l'effet du genre communicationnel de l'oral préparé et du type d'événement de communication.

Pour les contraintes extralinguistiques sur la variation, comme l'a montré l'analyse multivariée des données combinées de la France et du Québec, la diatopie influence la sélection des variantes exprimant la référence au futur, le futur synthétique étant plus fréquent en France qu'au Québec. Il y aurait lieu, afin d'approfondir cette dimension, d'inclure nos données provenant d'autres communautés francophones dans les recherches ultérieures. Le futur synthétique ressort comme le grand favori dans les deux communautés étudiées, suivi du présent, une variante souvent négligée dans les analyses, et du futur analytique qui arrive en dernier. Ceci tranche nettement avec la situation de l'oral spontané où le futur analytique gagne du terrain au détriment du futur synthétique. Le fait que la variante du présent soit aussi représentée montre également l'importance de ne pas négliger cette forme dans les analyses de la variation dans l'expression du futur en français contemporain. La prise en compte du groupe de facteurs lié au genre n'est pas aussi concluante puisque non seulement cet effet ne se dégage pas de l'analyse d'ensemble, mais lorsqu'il est sélectionné dans les analyses sur les communautés individuelles, son effet va 
dans des directions opposées. Cette situation pourrait s'expliquer par le faible nombre de locuteurs impliqués dans les analyses communautaires.

Pour les contraintes linguistiques, l'effet du type de verbe s'avère très net. Par ailleurs, l'absence de l'effet de la polarité sur la variation, tant dans l'analyse d'ensemble que dans les analyses communautaires, étonne et démarque nettement l'oral spontané et l'oral préparé. Ceci nous amène à conclure que les bulletins météo se rapprochent davantage de la norme de référence et que, dans le genre de discours associé à cet événement de communication, le futur synthétique n'est pas uniquement la chasse-gardée des énoncés négatifs mais s'emploie de manière productive dans plusieurs autres contextes linguistiques. L'analyse du corpus a donc indiqué que l'expression de la référence temporelle au futur dans les bulletins météo diffère sensiblement de ce qui s'observe dans l'oral spontané, tant sur le plan de la distribution des variantes que sur celui des contraintes pesant sur la variation.

En somme, le français employé dans les bulletins météo se rapproche de la norme associée au français de référence en raison de la large présence du futur synthétique dans plusieurs contextes linguistiques, comme par exemple les 40 articles de presse écrite étudiés par Waugh et Bahloul (1996) dans lesquels le FS domine largement (79\% des références au futur contre $21 \%$ pour le FA). Ici, ce ne sont pas tant des facteurs sociaux qui motivent l'usage de cette forme mais plutôt le registre, une dimension déjà explorée par Mougeon et al. (2010). Par ailleurs, le français des bulletins météo se distancie de l'oral spontané par la faible présence du futur analytique, en dépit du fait que la majorité des données traitent d'un futur relativement proche, un aspect qui devrait pourtant favoriser cette variante. Enfin, l'expression de la référence temporelle au futur dans les bulletins 
météo se démarque aussi par la forte représentation du présent, une forme souvent considérée périphérique. Nous interprétons ce résultat en fonction de la situation relative à l'événement de communication dans lequel la référence temporelle au futur s'avère implicite, ce qui pourrait rendre redondante la marque morphologique verbale.

L'analyse du français des bulletins météo constitue un bon exemple de la prise en compte d'un autre genre de discours, en l'occurrence ici l'oral préparé, pour mieux comprendre la dynamique concurrentielle des formes verbales de l'expression temporelle du futur en français contemporain. À ce titre, il serait souhaitable dans les études ultérieures d'examiner d'autres genres, entre autres celui représenté par la communication médiée par ordinateurs (Starke 2015), également marquée par l'hybridité des canaux oral et écrit (Labeau 2014, Blondeau, Tremblay et Drouin 2014). Notre analyse a bien montré la nécessité de se dégager d'une approche centrée principalement sur l'oral conversationnel et d'explorer de multiples genres discursifs pour l'étude de la variation avant de conclure à la mort éventuelle du futur synthétique dans le français d'aujourd'hui ou... du futur.

\section{Références}

Antonini, Annibale. 1753. Principes de la grammaire françoise, pratique et raisonnée. Paris : Duchesne.

Arnaud, Antoine et Claude Lancelot. 1660. Grammaire générale et raisonnée. Paris : P. LePetit.

Bahloul, Maher et Linda Waugh. 1996. La différence entre le futur simple et le futur périphrastique dans le discours journalistique. Modèles Linguistiques 33:19-36. 
Blondeau, Hélène. 2006. La trajectoire de l'emploi du futur chez une cohorte de Montréalais francophones entre 1971 et 1975. Revue de l’Université de Moncton 37:73-98.

Blondeau, Hélène, Dion, Nathalie et Zoe Ziliak Michel. 2014. Future temporal reference in the bilingual repertoire of Anglo-Montrealers: A twin variable. International Journal of Bilingualism (published online first 15 January 2013, 10.1177/1367006912471090), 18:674-692.

Blondeau, Hélène, Tremblay, Mireille et Patrick Drouin. 2014. Hybridité et variation dans les SMS: Le corpus Texto4Science et l'oralité en français montréalais. The Canadian Journal of Linguistics / La revue canadienne de linguistique 591:137165

Brunot, Ferdinand. 1966. Histoire de la langue française, Tome VI, 2ème partie. Paris : Éditions Armand Colin.

Chevalier, Gisèle. 1994. L'emploi des formes du futur dans le parler acadien du sud-est du Nouveau-Brunswick. Dans Les Acadiens et leur(s) langue(s) : quand le français est minoritaire, sous la direction de Lise Dubois et Annette Boudreau, 75-89. Moncton : CRLA, Université de Moncton.

Confais, Jean-Paul. 1995. Temps-mode-aspect: les approches des morphèmes verbaux et leurs problèmes à l'exemple du français et de l'allemand, $2^{\mathrm{e}}$ éd. revue et augmentée. Toulouse : Presses universitaires du Mirail

$\begin{array}{llll}\text { temporal } & \text { reference } & \text { in }\end{array}$




\section{septembre 2014.C}

Deshaies Denise et Eve Laforge. 1981. Le futur simple et le futur proche dans le franç ais parlé. Langues et linguistique 7:21-37.

Emirkanian, Louisette et David Sankoff. 1985. Le futur simple et le futur périphrastique dans le français parlé, Actes du XVII congrès international de linguistique et philologie romanes - Morphosyntaxe des langues romanes. Vol. 4:397-407.

Fleischman, Suzanne 1982. The future in thought and language: Diachronic evidence from Romance. Cambridge : Cambridge University Press.

Fournier, Jean-Michel. 2001. L'analyse du présent dans les grammaires de l'âge classique. Cahiers Chronos 7:1-26.

Gougenheim, Georges 1929 / 1971. Etudes sur les périphrases verbales de la langue française. Paris : Nizet.

Grimm, Rick .D. 2010. A Real-time study of Future Temporal Reference in Spoken Ontarian French, University of Pennsylvania Working Papers in Linguistics, vol. 16:83-92.

Halmøy, Odile. 1993. Futur en ra / futur en va: distribution en contexte. Dans Les langues menacées : actes du XVe Congrès international des linguistes, sous la direction de André Crochetière, Jean-Claude Boulanger et Conrad Ouellon, 75-78. Sainte-Foy : Les Presses de l'Université Laval.

Imbs, Paul. 1968. L'emploi des temps verbaux en français moderne: Essai de grammaire descriptive. Paris : Klincksieck.
Commented [Philip Co13]: L’article a été publié dans la revue, alors vous pourriez citer la version publiée (avec les pages). Voici la référence : Journal of French Language Studies 25(3): 339-365.
Commented [Philip Co14]: Je pense que c'est 1992, surtout si vous regardez à Lesage et Gagnon ci-dessous. 
James, Francis. 2014. Naissance d’une spécialité télévisuelle: la présentation de la météo.

Disponible sur: http://industrie-culturelle.fr/industrie-culturelle/naissance-

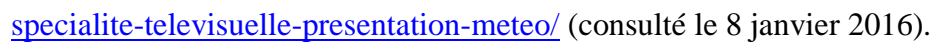

Jeanjean, Colette. 1988. Le futur simple et le futur périphrastique en français parlé. Dans Grammaire et histoire de la grammaire. Hommage à la mémoire de Jean Stefanini, sous la direction de Claire Blanche-Benveniste, André Cheurel et Maurice Gross, 235-257. Provence : L’Université de Provence.

King Ruth et Terry Nadasdi. 2003. Back to the future in Acadian French. Journal of French Language Studies 13:323-337.

Labeau, Emmanuelle. 2008. « Il y a une éternité, il y a un siècle, il y an un an... A quoi sert le PS dans les discours de voeux?' ». Dans Congrès Mondial de Linguistique Française - $C M L F^{\prime} 08$, sous la direction de Jacques Durand, Benoît Habert et Bernard Laks. Paris : Institut de Linguistique Française. Disponible en ligne : http://www.linguistiquefrancaise.org/index.php?option=article\&access=standard\&I $\underline{\text { temid=129\&url=/articles/cmlf/pdf/2008/01/cmlf08144.pdf }}$

Labeau, Emmanuelle. 2012. Time, Tense and Weather: Variation of temps in Francophone weather bulletins. Communication présentée au colloque, Regional Varieties, Language Shift and Linguistic Identities, Aston University, Birmingham.

Labeau, Emmanuelle. 2014. Quand l'analytique se fait synthétique: les formes verbales périphrastiques dans le texto, Studii de lingvistica 4:131-144.

Labeau, Emmanuelle. 2015. Il était une fois le passé simple.... Journal of French Language Studies 25:165-187. 
Labeau, Emmanuelle et Hélène Blondeau. 2015. 'La référence temporelle au futur dans les bulletins météo: approche diatopique francophone'. Communication présentée à la Conférence annuelle de l'Association for French Language Studies, Caen, France.

Labeau, Emmanuelle et Thomas Holyoak. 2007. Le présent suffit-il à faire 1'histoire ?, Revue Romane 42:167-312.

Leblanc, Carmen. 2011. Du conditionnel dans les propositions en si: Étude de la variation morphosyntaxique dans le français de la capitale nationale du Canada. Sarrebruck : Éditions universitaires européennes.

Lesage, René. 1991. Notes sur l'emploi du présent à valeur de futur dans les quotidiens québécois. Revue québécoise de linguistique théorique et appliquée 10:117-131.

Lesage, René et Sylvie Gagnon, Sylvie. 1992. Futur simple et futur périphrastique dans la presse québécoise. Dans Les langues menacées : actes du XVe Congrès international des linguistes, sous la direction der André Crochetière, Jean-Claude Boulanger et Conrad Ouellon, 367-370. Sainte-Foy : Les Presses de l'Université Laval.

Lindschouw, Jan. 2010. Variation et évolution du futur synthétique et du futur analytique du moyen français au français moderne. Communication présentée au colloque Diachro- $V$, Lyon, France.

Mougeon, Raymond, Terry Nadasdi et Katherine Rehner 2010. The sociolinguistic competence of immersion students. Toronto : Multilingual Matters.

Poplack, Shana. 2001. Variability, frequency and productivity in the irrealis domain of French. Dans 
Frequency and the emergence of linguistic structure, sous la direction deJoan Bybee et Paul Hopper, 405-428. Amsterdam : John Benjamins.

Poplack, Shana et Nathalie Dion. 2009. Prescription vs. praxis: The evolution of future reference in French. Language 85:557-587.

Poplack, Shana et Danielle Turpin 1999. Does the future have a future in (Canadian) French ? Probus, 11:134-164.

Poplack, Shana, Allison Lealess et Nathalie Dion. 2013. The evolving grammar of the French subjunctive. Probus 25:139-195.

Sankoff, David, Sali A. Tagliamonte et Eric Smith. 2005. Goldvarb X: A variable rule application for Macintosh and Windows. http://recombcg.uottawa.ca/lab/software.html

Söll, Ludwig. 1983. De la concurrence du futur simple et du futur proche en français moderne. Dans Etudes de grammaire française descriptive, sous la direction de Franz-Josef Hausmann, 16-24. Heidelberg : Julius Groos Verlag.

Starke, Elizabeth 2015. Caractéristiques du français non-standard — ce qu'on croit, et ce qu'on voit dans les données. Conférence plénière au colloque Association for French Language Studies, Caen, France.

Sundell, Lars-Goran. 1991. Le temps futur en français moderne. Uppsala : Acta Universitatis upsaliensis.

Tagliamonte, Sali A. 2006. Analysing sociolinguistic variation. Cambridge : Cambridge University Press.

Vet, Co 1993. Conditions d'emploi et interprétation des temps futurs en français. Verbum 4 (les aspects dans le discours narratif):71-84. 
Wagner, Suzanne Evans et Gillian Sankoff. 2011. Age grading in the Montréal French inflected future. Language Variation and Change 23:275-313

Wales, Mavis Lynn. 2002. The relative frequency of the synthetic and composite futures in the newspaper Ouest-France and some observations on distribution. Journal of French Language Studies 12:73-93.

Waugh, Linda et Bahloul, Maher 1996. La différence entre le futur simple et le futur périphrastique dans le discours journalistique. Modèles Linguistiques 17:19-36.

Wilmet, Marc. 1970. Le système de l'indicatif en moyen français. Genève : Droz.

Zimmer, Dagmar. 1994. Ça va tu marcher, ça marchera tu pas, je le sais pas (71:15) Le futur simple et le futur périphrastique dans le français parlé à Montréal. Langues et linguistique 20:213-226. 